For Internal Distribution Only

Accelerator Division

Alternating Gradient Synchrotron Department BROOKHAVEN NATIONAL LABORATORY

Upton, New York 11973

Accelerator Division

Technical Note

AGS/AD/Tech. Note No. 469

Emittance Blow-up from A20 Multi-wire in the AGS

M. J. Syphers

July 22, 1997 


\title{
Emittance Blow-up from A20 Multi-wire in the AGS
}

\author{
M. J. Syphers
}

August 4, 1997

The multi-wire beam profile monitor at A.20 in the AGS was installed to be able to look at several-turn (1 to 2 passes) beam profiles upon injection to ascertain amplitude function mismatches between the BtA transfer line and the AGS. This instrument has also been used to attempt emittance measurements in the AGS by allowing the beam to pass through hundreds of turns in hopes of measuring the equilibrium beam emittance. This measurement produces results with emittances much larger than expected, and also induces severe beam loss over a few hundred turns. The results can be explained simply by multiple Coulomb scattering from the Tungsten wires in the profile monitor.

The A20 multi-wire[1] is composed of 64 vertical wires (for horizontal profile measurements) and 32 horizontal wires (for vertical measurements) of $0.1 \mathrm{~mm}$ diameter tungsten each separated by $2 \mathrm{~mm}$. A charged particle passing through one of these wires will be Coulomb scattered and the the trajectory in one transverse plane will be altered by a typical amount

$$
\Delta \theta_{r m s}=\frac{13.6 \mathrm{MeV}}{p v} z \sqrt{\frac{\ell}{L_{r a d}}}
$$

where $\ell$ is the thickness of the wire, $L_{\text {rad }}$ is the radiation length of the wire material, $z$ is the charge number, and $p$ and $v$ are the particle's momentum and speed. For this report, we will consider the particles to be protons at AGS injection, for which $\Delta \theta_{r m s}$ is approximately $1 \mathrm{mrad}$ for $0.1 \mathrm{~mm}$ thick tungsten.

As an example, at the location of the multi-wire, the value of the vertical amplitude function is approximately $11 \mathrm{~m}$, and so a $1 \mathrm{mrad}$ angular deflection will induce a betatron oscillation whose amplitude at a $\beta_{\max }$ location would be $\sqrt{\beta_{\min } \beta_{\max }} \Delta \theta=$ $15 \mathrm{~mm}$. If the incoming emittance was $15 \pi \mathrm{mm}-\mathrm{mrad}$, then this $15 \mathrm{~mm}$ deflection would be compared to an rms beam size of only $\sqrt{15 \cdot 22 /(6 \cdot 2.4)}=4.8 \mathrm{~mm}$ !

For 1- or 2-pass measurements, the scattering is not much of a problem since the wires only cover about $5 \%$ of the beam. However, if the beam is allowed to pass through the multi-wires time and again, then after about 100 turns each particle will 
Wire thickness $=0.1 \mathrm{~mm}$; spacing $=2 \mathrm{~mm}$

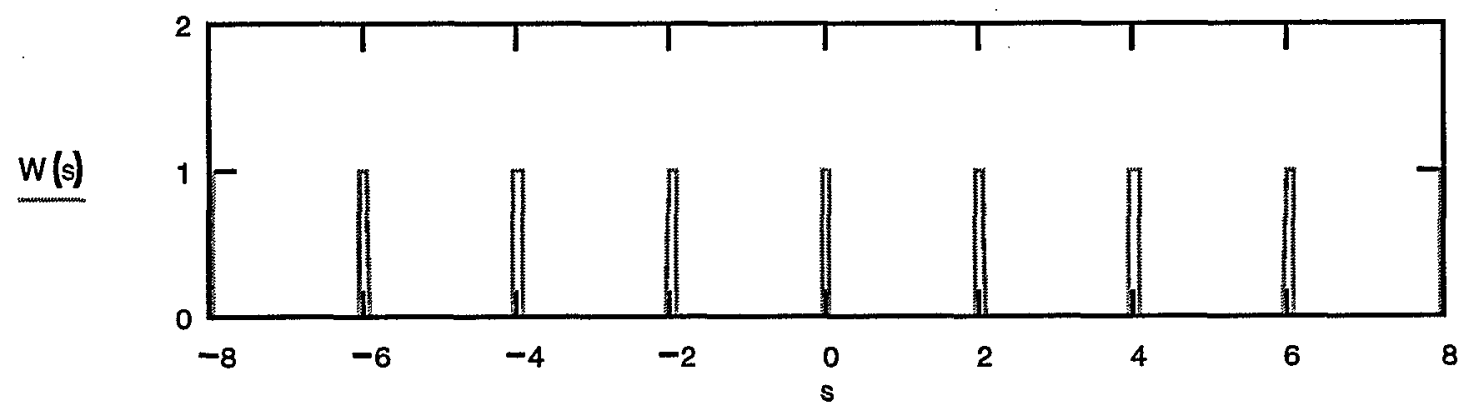

Figure 1: The function $W(x)$ describing the transverse wire positions.

have passed through a wire about 5 times, at which time it's amplitude would have grown to roughly $15 \mathrm{~mm} \cdot \sqrt{5}=34 \mathrm{~mm}$, and thus it would quickly leave the AGS aperture. The profile given by the A20 instrument is the integrated signal of all the passages of the beam.

To model these conditions, a short program was written to track particles around the AGS with scattering in the wires included. The function $W(x)$ describes the locations of the wires, taking on a value of 1 if the particle's transverse position is within a wire, and 0 otherwise, as shown in Fig. 1 . Then, the phase space trajectory of the $i$-th particle on the $n+1$-st turn is mapped by

$$
\left(\begin{array}{c}
x_{i, n+1} \\
p_{i, n+1}
\end{array}\right)=\left(\begin{array}{cc}
\cos 2 \pi \nu & \sin 2 \pi \nu \\
-\sin 2 \pi \nu & \cos 2 \pi \nu
\end{array}\right)\left(\begin{array}{c}
x_{i, n} \\
p_{i, n}+W\left(x_{i, n}\right) \cdot \beta \Delta \theta_{n}
\end{array}\right)
$$

where here, $p \equiv \alpha x+\beta x^{\prime}$, and $\Delta \theta$ is a normally distributed random variable with rms value $\Delta \theta_{r m s}$. On each passage of the wires, if the amplitude of the particle becomes greater than $25 \mathrm{~mm}$, the particle is considered lost. At the conclusion of the tracking, the positions of all particles - until they are lost - are summed up to give the integrated profile.

Fig. 2 shows the result of tracking a Gaussian distribution of particles for 400 turns without the effects of the wires. The left plot shows the integrated profile over the 400 turns. The particles survive and maintain their initial emittance of $15 \pi \mathrm{mm}$ $\mathrm{mrad}$. With the wires in place, the same simulation gives the profile and intensity plot shown in Fig. 3. The beam is almost completely lost within about 400 turns. This final integrated profile is to be compared with the actual profile measurement shown in Fig. 4, which was taken under similar beam conditions.

A rough fit to a Gaussian of the central core of the beam is indicated in Fig. 3. "Tails" are evident in the final distribution, just as seen in the measurements, and 

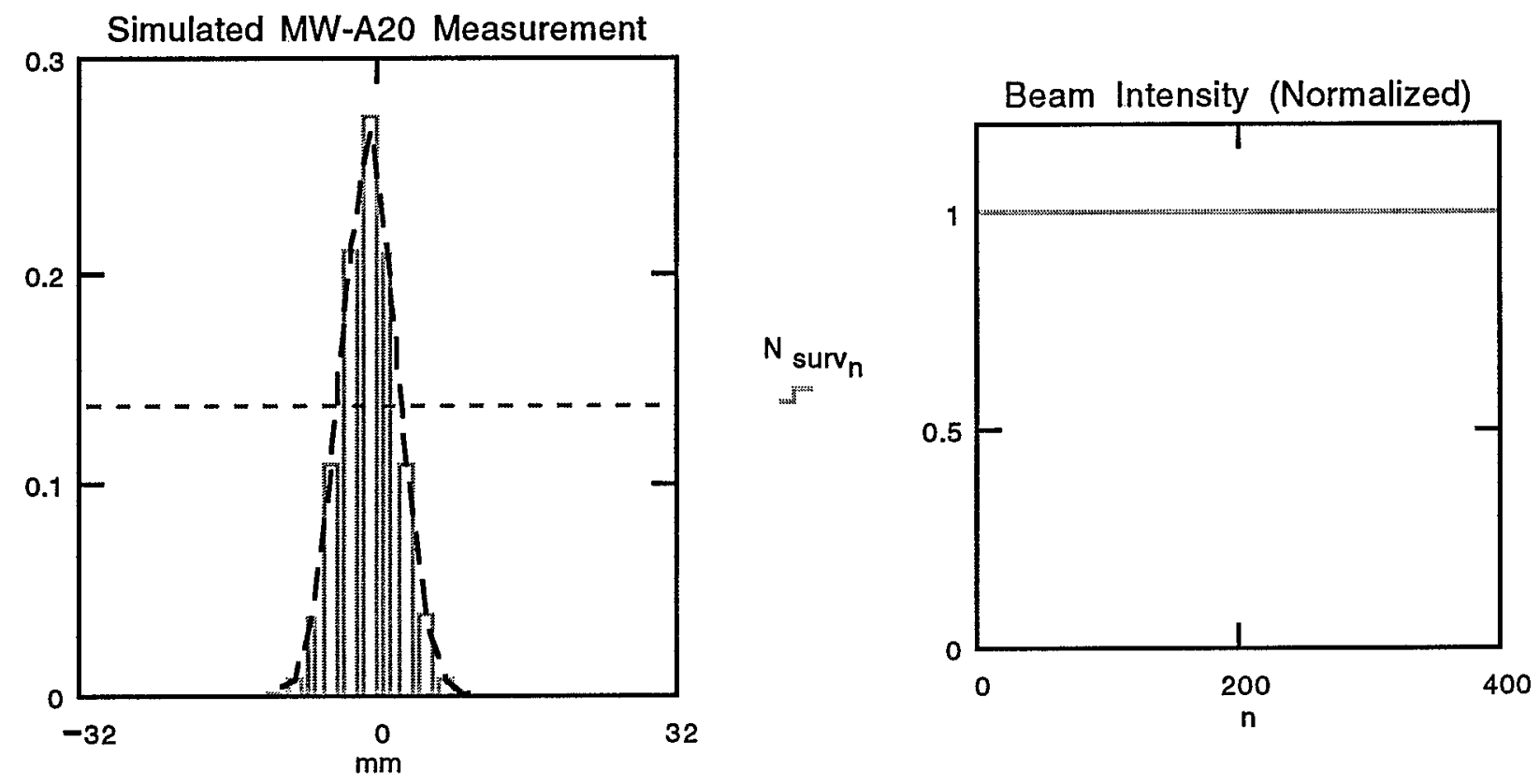

Figure 2: Results without multi-wires present.
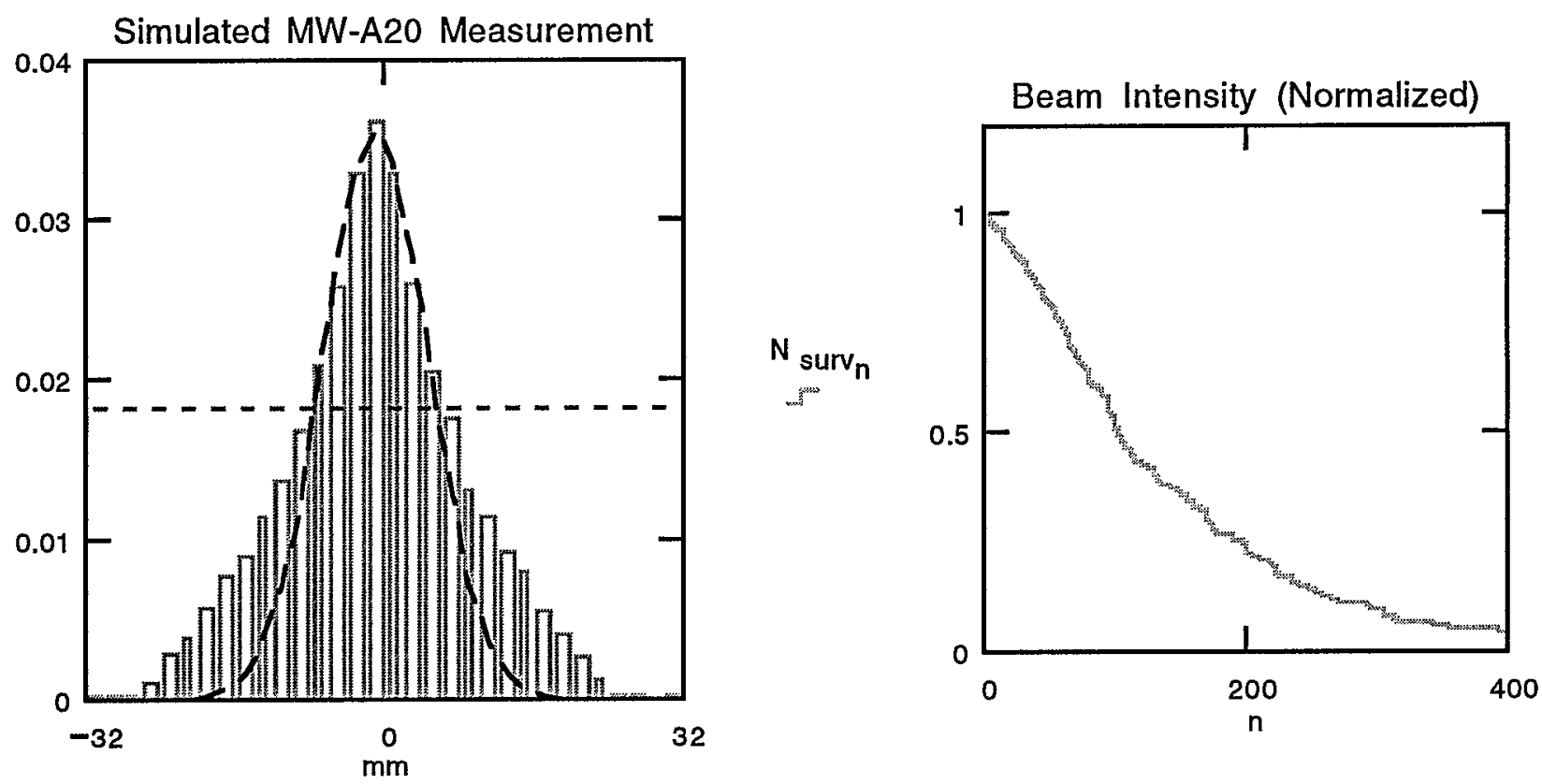

Figure 3: Results with the multi-wires present. 


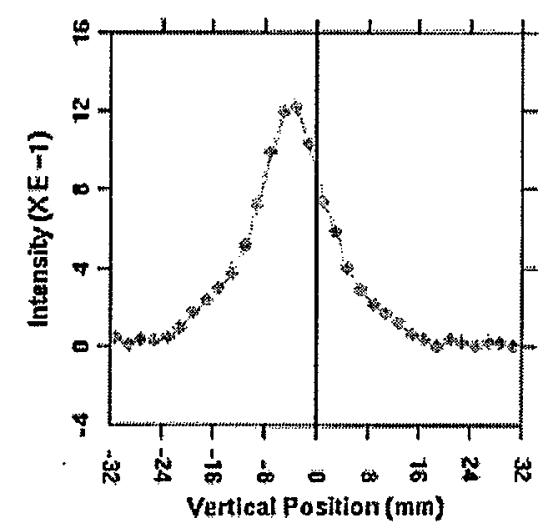

Figure 4: Beam profile measurement with the A20 multi-wires. The beam circulated the AGS until all particles were lost, which occurred after a few hundred turns. The emittance determined from other single-pass multi-wire measurements in the BtA line was about $15 \pi$ mm-mrad.

the rms of the total distribution gives an emittance of $130 \pi \mathrm{mm}$-mrad. The size of the central core, however, corresponds to an emittance of about $40 \pi \mathrm{mm}-\mathrm{mrad}$.

The program was used to verify that after only one or two passes through the multi-wires, no emittance blow-up could be detected from the particle distributions. Thus, the tungsten multi-wire system is adequate for its intended use.

\section{References}

[1] H. Huang, et al., "A Multi-wire Beam Profile Monitor in the AGS," proc. 1997 Part. Accel. Conf., Vancouver, in press. 
$8 / 7197$

Tech. Note No. 468

not done 